\title{
Exploring the Development Requirements for Virtual Reality Gait Analysis
}

\author{
Mohammed Soheeb Khan ${ }^{1}$, Vassilis Charissis ${ }^{1}$ and Sophia Sakellariou ${ }^{2, *}$ \\ 1 School of Computing, Engineering and Built Environment, Glasgow Caledonian University, \\ Glasgow G4-0BA, UK; Soheeb.Khan@gcu.ac.uk (M.S.K.); Vassilis.Charissis@gcu.ac.uk (V.C.) \\ 2 Department of Radiology, Queen Elizabeth University Hospital, Glasgow G51-4TF, UK \\ * Correspondence: sophia.sakellariou@ggc.scot.nhs.uk
}

Received: 12 February 2019; Accepted: 4 April 2019; Published: 10 April 2019

check for updates

\begin{abstract}
The hip joint is highly prone to traumatic and degenerative pathologies resulting in irregular locomotion. Monitoring and treatment depend on high-end technology facilities requiring physician and patient co-location, thus limiting access to specialist monitoring and treatment for populations living in rural and remote locations. Telemedicine offers an alternative means of monitoring, negating the need for patient physical presence. In addition, emerging technologies, such as virtual reality (VR) and immersive technologies, offer potential future solutions through virtual presence, where the patient and health professional can meet in a virtual environment (a virtual clinic). To this end, a prototype asynchronous telemedicine VR gait analysis system was designed, aiming to transfer a full clinical facility within the patients' local proximity. The proposed system employs cost-effective alternative motion capture combined with the system's immersive 3D virtual gait analysis clinic. The user interface and the tools in the application offer health professionals asynchronous, objective, and subjective analyses. This paper investigates the requirements for the design of such a system and discusses preliminary comparative data of its performance evaluation against a high-fidelity gait analysis clinical application.
\end{abstract}

Keywords: virtual reality; telemedicine; gait analysis; motion capture; locomotion

\section{Introduction}

Advances in modern medicine and better quality of life have resulted in higher life expectancies in the developed world. The population aged 65 years and over is projected to grow by around $50 \%$ over the next 23 years in the UK [1]. The rural areas, in particular, have seen a larger increase in average age than urban areas [2]. Additionally, internal migration statistics have shown an increase in population moving into rural locations [3]. Such population migration trends present a number of challenges for the future, in particular regarding access to modern health services.

Abnormal gait and locomotion deformities can be a result of various causes. Ageing, disease, and accidental injuries are all common factors. The probability of a gait-related issue increases by $10 \%$ between ages $60-69$ and by $60 \%$ over the age of 80 years [4]. Observation of locomotion is a complex study involving internal and external factors, which can result in a variety of positional changes $[5,6]$. Typically, the diagnostic triage of such injuries and pathologies requires the physical presence of the subject for orthopaedic and locomotor examination. Vital clues for diagnosis can be obtained from visual observations by trained professionals. Indications such as swellings and bruising can ultimately aid in determining the cause and result in a diagnosis. However, long-term patients with locomotion-affected pathologies, acute and chronic pathologies, such as osteoarthritis can be required to visit clinics routinely. 
This could involve such patients having their gait assessed over a period of time. Such gait-related observation utilises the use of a motion-capture system to record the patient's movement to be analysed in the specialised motion analysis software. Gait analysis facilities enable the acquisition of high-quality, quantifiable biomechanics data that are analysed objectively [7-10]. However, such facilities are very limited, are mostly situated in major cities, and require substantial space and costs to operate. Furthermore, concurrent global financial issues, limited resources, and the constantly growing population have prompted interest from various governing bodies to reform and seek alternative methods for delivering high-quality healthcare [11,12]. The advancements on sensors, 3D visualisation and the increase of communication innovations have made telemedicine a promising solution for many issues faced by the current healthcare systems $[13,14]$. To this end, telemedicine-enabled VR gait analysis systems could offer a viable alternative, as presented in previous preliminary studies $[15,16]$.

Adhering to the above, the aim of this work is to identify a precise and financially viable rehabilitation alternative, which could be deployed in a small footprint room (i.e., an average house bedroom). As such, we propose a novel system that utilises advanced 3D/VR visualization, motion tracking sensors, and remote data analysis for the acquisition, presentation, and analysis of motion data derived by the patient. As two of the major restrictions are the cost and space of current rehabilitation facilities, our system employs cost-effective motion-capture, built from off-the-shelf equipment, to be utilised with our immersive 3D virtual gait analysis clinic. The latter enables the collection of data related to biomechanics independently. In turn, the data are mapped on to a unique 3D anatomical data set, which is then visualised in the 3D virtual gait analysis clinic application. This paper presents a pilot study designed to gauge subjective responses and user experience feedback by ten participants, which used the prototype system in this experimental form.

The following section presents the musculoskeletal issues that require gait analysis and rehabilitation systems. In turn, the paper discusses the contemporary state of the art facilities and the issues that require an alternative approach to the solution. Technological advances and equipment are also discussed in detail. Section 4 presents the proposed system solution, whilst Section 5 presents the evaluation process and the questionnaire used to acquire the subjective feedback form the participants. The paper concludes with the overview of the work undertaken and the results derived during the evaluation. Finally, the paper presents a tentative plan of work based on the received feedback and recorded users' experience of the proposed VR gait analysis system.

\section{Musculoskeletal (MSK) Issues Requiring Gait Analysis}

\subsection{Gait Analysis}

Gait analysis is described as the analysis of the walking pattern produced during one's walk cycle. This particular type of human motion analysis is used to assess, plan, and treat individuals with conditions that affect their ability to walk. According to Kale et al., gait refers to the style of walking of an individual $[17,18]$. Assessment of gait is also commonly used to identify any abnormalities related to posture movement in people with deformities or musculoskeletal (MSK)-related injuries [18]. Previous studies have shown that gait tracking methods can be used for injury surveillance, rehabilitation, physical training, and therapy. They are effective methods for early intervention and diagnosis, which can lead to the prevention of further or permanent injury [19]. The musculoskeletal system is utilised profusely throughout the diverse phases of the walk cycle. Gait movement is specific for each individual and can be assessed by analysing one's gait cycle. Any disorder of bones and joints usually affects the gait. Natural changes in body proportions, growth, ageing, and pathological conditions can also change a person's gait.

Motion capture and motion tracking is the ability to record and track locomotion or the movement of an object from physical space into 3D virtual space [20]. Because of the versatility in applications of such systems, this technology is being utilised in various disciplines across many industries. Some of these include biomechanics, gait analysis, computer interaction, and computer animation. Motion 
capture technology enables accumulation of movement data and transferability of data into a 3D virtual space. The data can be remapped onto a 3D avatar, played back, analysed, and modified synchronously (real-time) or asynchronously (store and forward).

The technological innovations in motion-capture systems have made it possible to acquire and collect complex motion data for biomechanics of MSK structures [21,22]. Motion capture techniques are being deployed and implemented across a range of disciplines. They are predominantly used in the entertainment industry such as animation, film, and videogames. The accuracy of depiction and transferability of motion-capture data created a new research area for clinicians and other medical-related professions to utilise this technology for gait analysis and the study of kinematics [7-9].

\subsection{Gait Cycle}

The gait cycle is defined as the time during which a complete set of walking sequences takes place. By understanding each of the different events of a typical walk cycle, practitioners can assess, measure, and compare gait. A gait cycle begins with heel contact of either foot and ends with heel contact of the same foot; therefore, one gait cycle consists of two steps, one of the right foot and of the left foot. The gait cycle has been broken into two phases, "stance phase" and "swing phase". Stance phase comprises $60 \%$ of the walk cycle whilst the swing phase comprises the other $40 \%$, as illustrated in Figure 1 . The stance phase includes loading response, mid stance, terminal stance, and pre-swing, which lasts from the initial contact to contact toe-off. The swing phase includes initial swing, mid swing, and terminal swing, which lasts from toe-off to the next initial contact [23]. The major events during the gait cycle include initial contact, opposite toe-off, heel rise, opposite initial contact, toe-off, feet adjacent, tibia vertical, and then back to initial contact.

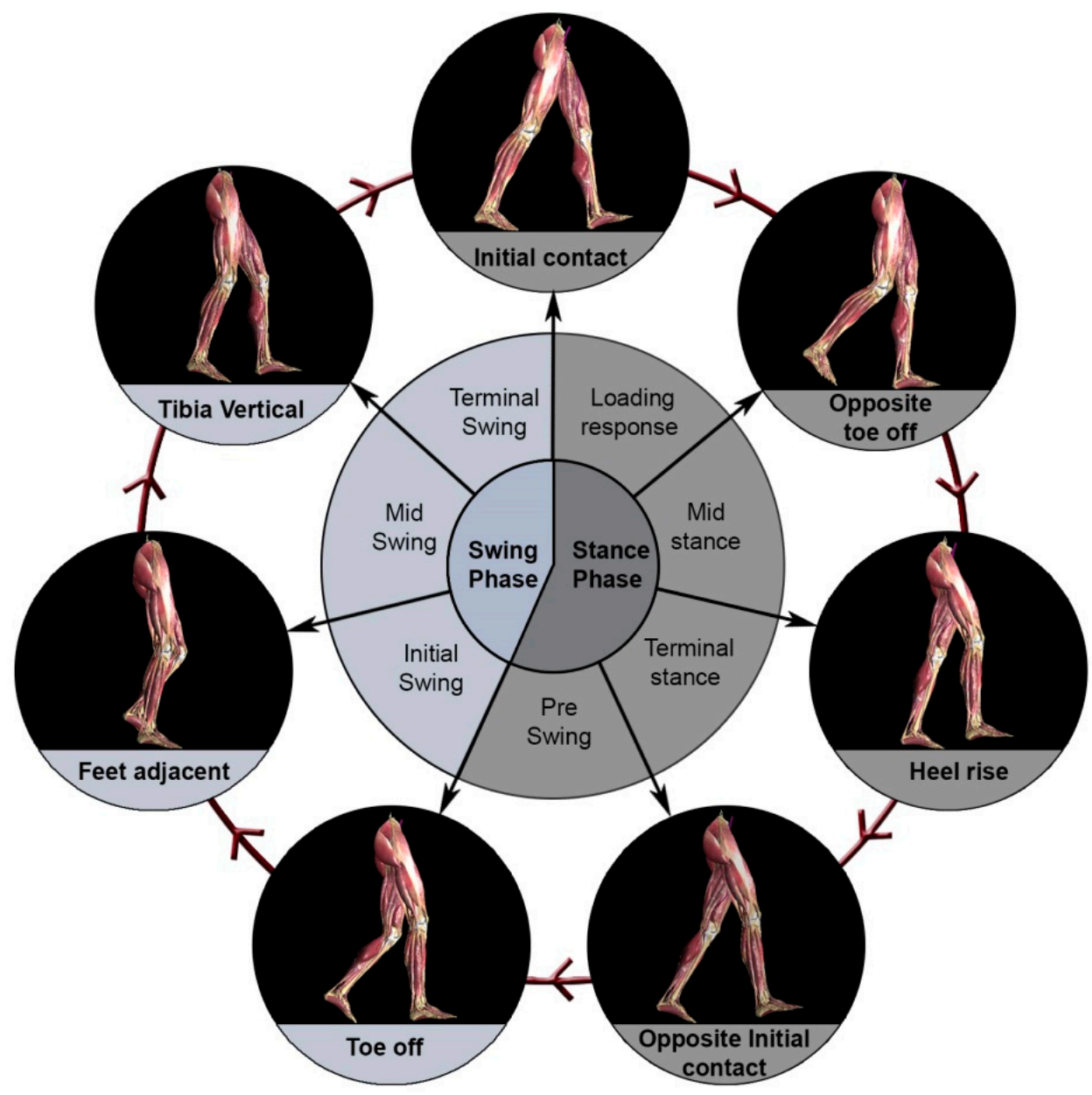

Figure 1. Different positions of the leg during a single gait cycle. 


\subsection{Abnormal Gait}

During the walking phase, when both feet are in contact with the floor simultaneously, this is referred to as "double stance". Stride length is the distance between the two successive placements of the same foot [17]. One stride is made up of two steps, left and right, each of which is the distance that the named foot moves forward in front of the other one. If gait is pathological, the stride length can vary, for instance, one foot may hop and the other may be in the air.

Abnormal gait can be commonly caused by pathologies and injuries related to the musculoskeletal structure, the brain, and/or the spinal cord. This could be temporary due to injury, acute pathology, and even long-term diseases inherited or degenerative. The National Center for Biotechnology Information [24] details some common causes affecting gait as:

- Accidental damage and injuries (fractures, tendon ruptures, tendonitis, muscle sprains)

- Arthritis (osteoarthritis, rheumatoid arthritis, septic arthritis)

- Congenital birth issues (Club foot, leg length inequality)

- Infection (myositis, inner ear infection, encephalitis)

- Psychological disorders

- Nervous system disorders (stroke, multiple sclerosis, cerebral palsy)

- Spinal cord pathologies (trauma, cord compression, nerve root compression, sciatica)

- Shin splints

- Skeletal abnormalities and side effects to procedures (scoliosis/kyphosis hip and knee replacements)

Abnormal gait is a common phenomenon that health professionals study, analyse, and treat. Much work has been done in this area, and common abnormal gaits have been identified, named, and categorised [24-26].

The hip is a complex musculoskeletal structure prone to injury as well as being affected by acute and chronic pathologies such as arthritis. Hip-related pathologies such as osteoarthritis often result in hip replacement [27], and diseases such as stroke can cause paralysis, leading to weakening of muscles and long-term complications with gait. Hip pain is a common area of interest for health professionals both in gait analysis and rheumatology. Aforementioned diseases and various other pathologies related to the hip require long term monitoring and rehabilitation of patients.

The hip joint is a ball and socket joint, which connects the lower limbs to the pelvis [28]. According to Moore, the hip joint is designed for stability over a wide range of movement. Furthermore, it is one of the most "movable" of all joints. The hip joint has three degrees of freedom and allows flexion/extension, abduction/adduction, and external/internal rotation [29].

Hip pathologies and treatment can have long-term effects on an individual's locomotion, limiting their movement [30,31]. Patients recovering from such pathologies may require rehabilitation over a long period of time. Such patients are in need of monitoring and need to have their gait assessed by experts regularly, to both monitor disease progression and/or treatment efficacy but also as an adjunct to personalizing rehabilitation efforts.

As previously mentioned, although gait analysis is clinically very useful, its user access and utilisation are severely limited by scarce resources such as space, expertise, and specialist kits (software and hardware), all of which are usually concentrated in large research centres. This results in the use of alternative, suboptimal monitoring techniques that create patient inequalities to service access.

The following section provides an overview of current methods of gait analysis and highlights the limitations of each with a view to defining the attributes of a complete cost-efficient, clinically acceptable new prototype solution. 


\section{Current Gait Analysis Systems and Research}

\subsection{Current Methods of Digital Gait Analysis}

Research suggests that contemporary gait analysis could be subdivided to gait research or clinical gait analysis [18]. The purpose of clinical gait analysis is to directly impact patient treatment, whereas gait analysis research impacts the discipline in general, contributing to the development of locomotion and kinematic studies.

Clinical gait analysis should either aid in diagnosis or management of the patient pathology or assist in patient rehabilitation and recovery. Therefore, clinical gait analysis needs to be utilised inpatient consultations. Previous research highlights limitations with current clinical gait analysis methodologies in assisting in the rehabilitating of patients [10] primarily relating to lack of time in the health professional-patient interaction. This in turns limits rehabilitation efforts to a single intervention without allowing experimentation and choice between different intervention techniques nor the facility to predict an outcome.

In comparison to clinical gait analysis, gait analysis research is less time-constrained, and deadlines for deliverable data are less prescriptive as they are separate to a clinical encounter. This allows researchers to spend more time with patients whilst trying different techniques and acquiring different sets of data. The findings from gait research are expanding the field generally as well as impacting clinical gait analysis.

Contemporarily, there are a variety of methods and techniques that are being utilised to carry out gait analysis. Some of these techniques are very simple and require simple patient and clinician contact, whilst other techniques employ additional sophisticated technologies to analyse patient motion. There are a host of factors that can be measured during gait analysis; some of these include velocity, step lengths, ground reaction forces, joint angles, distance travelled by patients, the maximum the patient can walk, and body posture. Muro-de-la-Herranhave suggested all these parameters can be categorised as the following two categories "Semi-subjective methods" or "Objective method" [32].

Semi-subjective methods involve the patient's gait being assessed by the clinician without utilising any sophisticated technology. This method relies more on specialist/health professional experience and observational skills. This simple form of gait analysis can be effective in determining patient limitations with locomotion (e.g., the time the patient takes from walking from point A to B). This methodology can be limiting as it is reliant on the health professional's visual diagnosis. However, more methodical motion analyses can be carried out by digitally recording the patient's gait and analysing it whilst replaying the recorded video at slower speeds.

Using video recording technology has a beneficial impact on the patient being consulted, as it limits the number of times they have to walk, in comparison to the clinician asking the patient to repeatedly perform the same movement. Although this approach can be utilised to understand and determine many parameters associated with gait, it is limited to only 2D observation and constrained to the single perspective the video was recorded from.

Objective gait analysis methods utilise a host of devices including advanced optical sensors, wearable and floor sensors, and floor and glass plates (Figure 2). These devices track and record various gait-specific parameters in high quality and can utilise specialised computer software to help with analysis of data, resulting in a more thorough and accurate analysis. This method limits subjectivity and reduces the chances of error. The data are collected, processed, and stored, and the information can be reproduced and compared to subsequent sets of data so as to monitor treatment outcomes. 


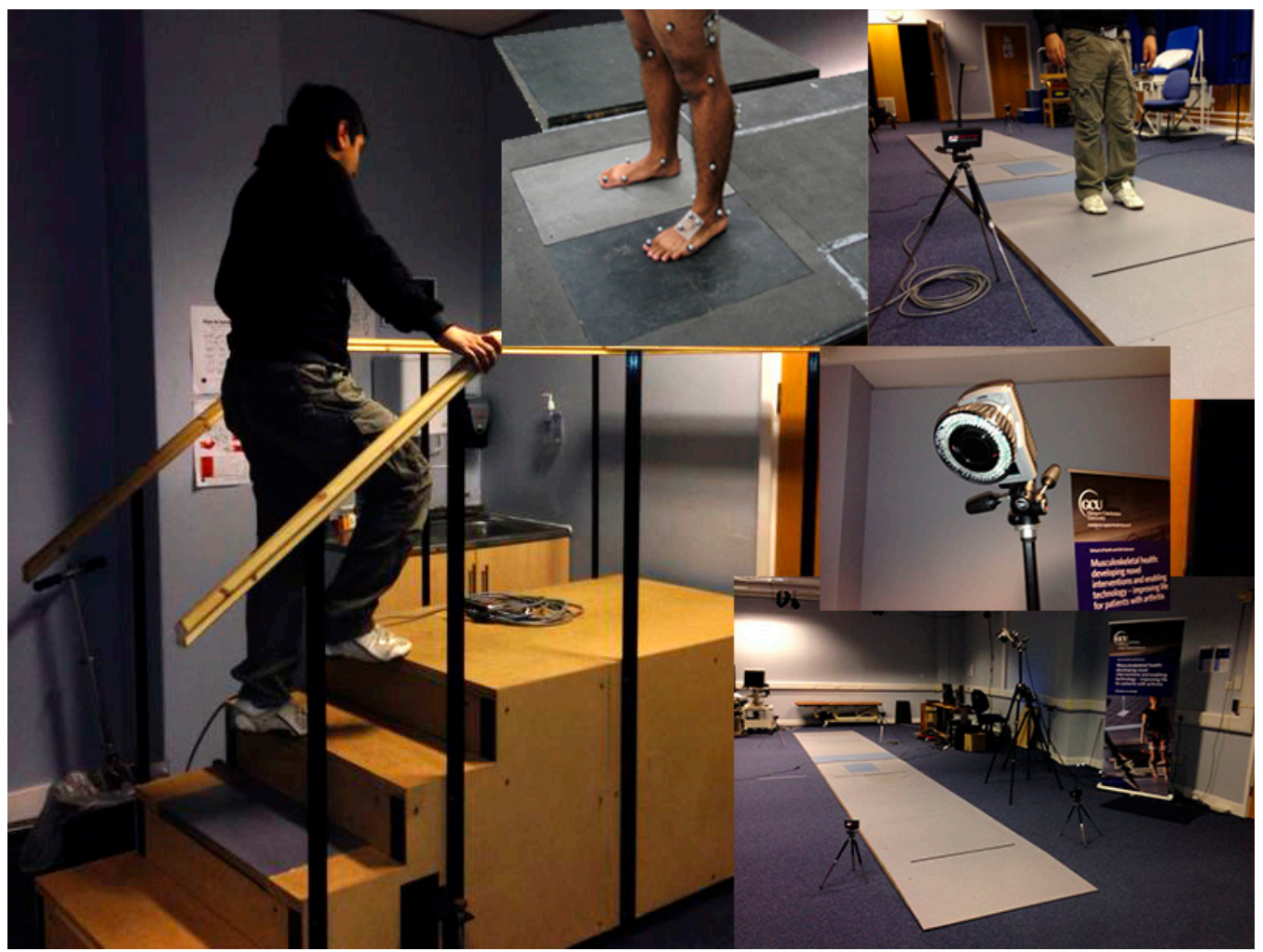

Figure 2. Different types of gait analysis equipment available at Glasgow Caledonian University (GCU) gait analysis laboratory [16].

\subsection{Motion Capture}

Notably, the historical progression of motion capturing technology is directly interlinked with the history of gait analysis [33]. Multiple methods and tools have been developed to record and track gait. Yet, motion capturing (Mo-Cap) systems are one of the most effective and accurate methods of recording gait for motion tracking. Such systems enable tracking of a moving object from the physical space into the 3D virtual space [21]. Developments in 3D graphics and Mo-Cap software make it possible for the retrieved data to be mapped onto any 3D avatar. This can then be played back, analysed, and modified synchronously (real-time) or asynchronously (store and forward).

The technological innovations in Mo-Cap systems have enabled the acquisition and collection of complex motion data for the biomechanics of musculoskeletal (MSK) structures [23,24]. The accuracy of depiction and transferability of motion-capture data created a new research area for clinicians and other medical-related professions to utilise this technology for gait analysis and the study of kinematics. Previous studies demonstrated that Mo-Cap for gait and motion analysis is remarkably accurate, and it contains both three-dimensional and temporal information in comparison to traditional methods for obtaining human mobility data. Adaptability and the customisation of such systems allow researchers and clinicians to focus their research specifically to the area that is of most interest to them, allowing the capturing of motion data related to selected MSK elements [34]. However, the majority of such systems require a large deployment footprint and multiple sensors positioned in the periphery in order to acquire any useful data, as depicted in Figure 3 below. This issue highlights the necessity of developing mobile and easily deployable systems that could operate in close proximity to the user/patient. 


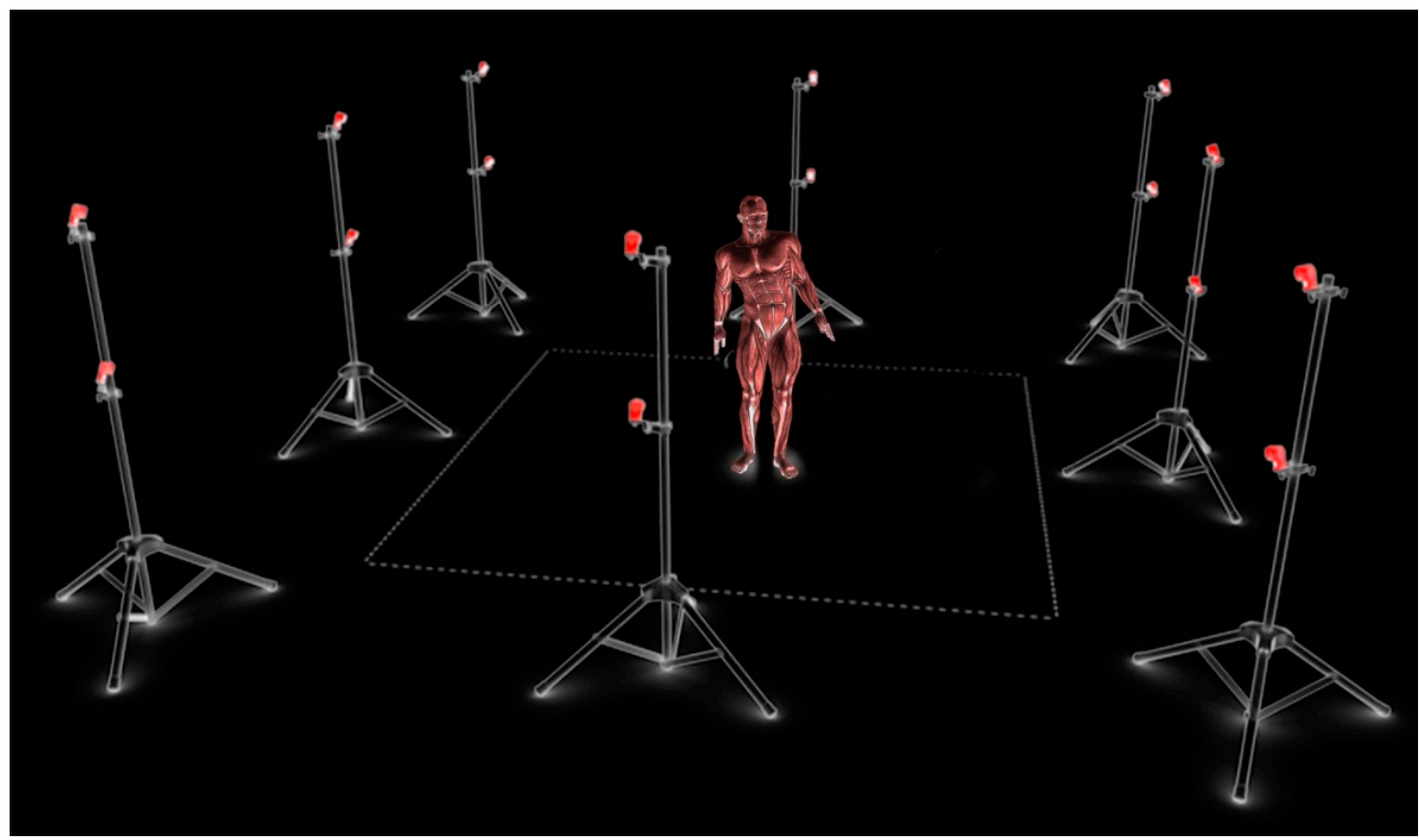

Figure 3. Visualisation of 3D virtual workspace generated from motion capture though several calibrated cameras in physical space.

\subsection{Motion Capturing and Rehabilitation System Trends}

Recent developments related to the videogame industry and associated peripheral devices have fueled researchers to begin development of cost-efficient, home-based telemedicine systems. Innovative inexpensive optical sensor technology designed to enhance videogame experiences have become an easily obtainable solution for multiple complex issues and desires for human-computer interaction (HCI) [35].

Clinicians and researchers have begun to experiment and develop tools with such devices to assist motion-driven tasks [34,36,37]. A sufficient amount of interest has been an aid for rehabilitation purposes $[37,38]$. A comparative study carried out by Stone and Skubic attempted to determine if low-budget systems such as Kinect (Microsoft ( $)$ ) and another web camera-based system could be utilised to predict falling vulnerability of the elderly in their home environment. They studied this by analysing captured data from both of the systems and compared it to the industry standard vicon systems [39]. Stone's study concluded both systems performed adequately, although the webcam-based system was more appropriate for their purposes. Upon analysing the study, it was observed that Stone's findings were limited because only one Kinect was employed in comparison to multiple cameras employed for the webcam-based systems. Therefore, the comparison test faced the aforementioned problems related to using single Kinects for Mo-Cap.

Most of the research concerning musculoskeletal pathology and anatomy with the usage of these alternative cost-effective solutions has been in view of rehabilitation purposes. Attygalle et al. developed a tool for a home-based rehabilitation setup, which incorporated the standard Biofeedback rehabilitation program [40]. In the latter work, a Wii (Nintendo () remote was utilised to successfully record data concerning rehabilitation for stroke patients. The data were analysed at a later stage (asynchronously) by therapists in order to track the patient's progress over the rehabilitation period. The utilization of off-the-shelf and inexpensive optical sensors for the development of cost-effective solutions for rehabilitation has been of intense interest in the last few years. A system proposed by Sundaresan and Chellappa made possible the construction of a completely articulated human body model [41]. This was implemented with the utilisation of video-recording from multiple web-cameras calibrated to work in tandem. The particular system enabled accurate tracking of the subject to be 
mapped on to the articulated model. A similar approach was investigated by Corazza et al., who used multiple webcams to create a visual hull from the silhouette of the subject to construct geometry [8]. Corcazza managed to track the gait of a subject and apply the motion to a virtual character. His work concluded that although the movement captured was satisfactory for some joints, much work was needed to obtain accurate results from other joints of the body for a complete gait cycle.

\subsection{Need for Remote Gait Analysis Motion Capturing Tools}

Current motion capturing systems are expensive. The system utilised by health professionals at Glasgow Caledonian University (GCU) costs approximately $£ 100,000$ for the equipment, and further costs include maintenance and software updates. Such systems need specialised setups, require a large space, and they need experts to operate the equipment. Gait analysis/diagnosis laboratories are most commonly situated at a designated facility that requires the patients to travel to and from routinely. The limited amounts of facilities have constrained the number of patients who can receive this triage. Patients face long waiting times prior to initial appointments, and people living in isolated and rural areas have limited or no access to this facility. This has created a need for tools that can educate and aid patients and health professionals to self-care remotely whilst still being monitored by specialised professionals asynchronously. Introduction of inexpensive videogame-based peripherals and hardware for tracking player motion have enticed researchers and hobbyists to begin experimenting with this technology (Microsoft Kinect (Microsoft $\odot$ )). This is discussed in more detail in the following section.

\section{Proposed System Solution}

Our system was developed through an iterative and incremental development process, which enabled the developing team to build and test the software application in multiple stages, aiming to eliminate major usability and functional issues. During system development, a number of specialist medical doctors were consulted for the accuracy of the system, regarding both the data collection capacity as well as the correct presentation of human anatomical and pathological data. Throughout this process, the project's aim was to enable health professionals and clinicians to interact efficiently and in a timely manner with the virtual dataset.

Additionally, the system was designed to offer a plethora of tools to the health professionals, to confidently analyse and monitor patients' gait idiosyncrasies and their rehabilitation progress. As such, the system could present in real-time the patient's motion in a VR environment and present simultaneously the related motion graphs. The latter could highlight instantly the "peak to peak" variations in the patient's motion pattern and enable the medical practitioner to adjust future rehabilitation exercises. The VR /3D model could be manipulated by both the patient and the medical practitioner in a 3D space, and it could be rotated or panned in order to present the best view of human body motion. The complete system is presented in action, capturing and analyzing a user's walking cycle, in Figure 4.

For the particular prototype system, it was deemed useful to employ a set of first-generation Microsoft Kinect sensors system, purely in order to prove the point that the hardware should not be a limitation or the major cost of such systems. A combination of Microsoft Kinect sensors was positioned diagonally in a room $(3 \times 4 \mathrm{~m})$. The rationale behind constraining the experimentation area was in order to resemble the typical size of a bedroom or a small office in a house environment. 


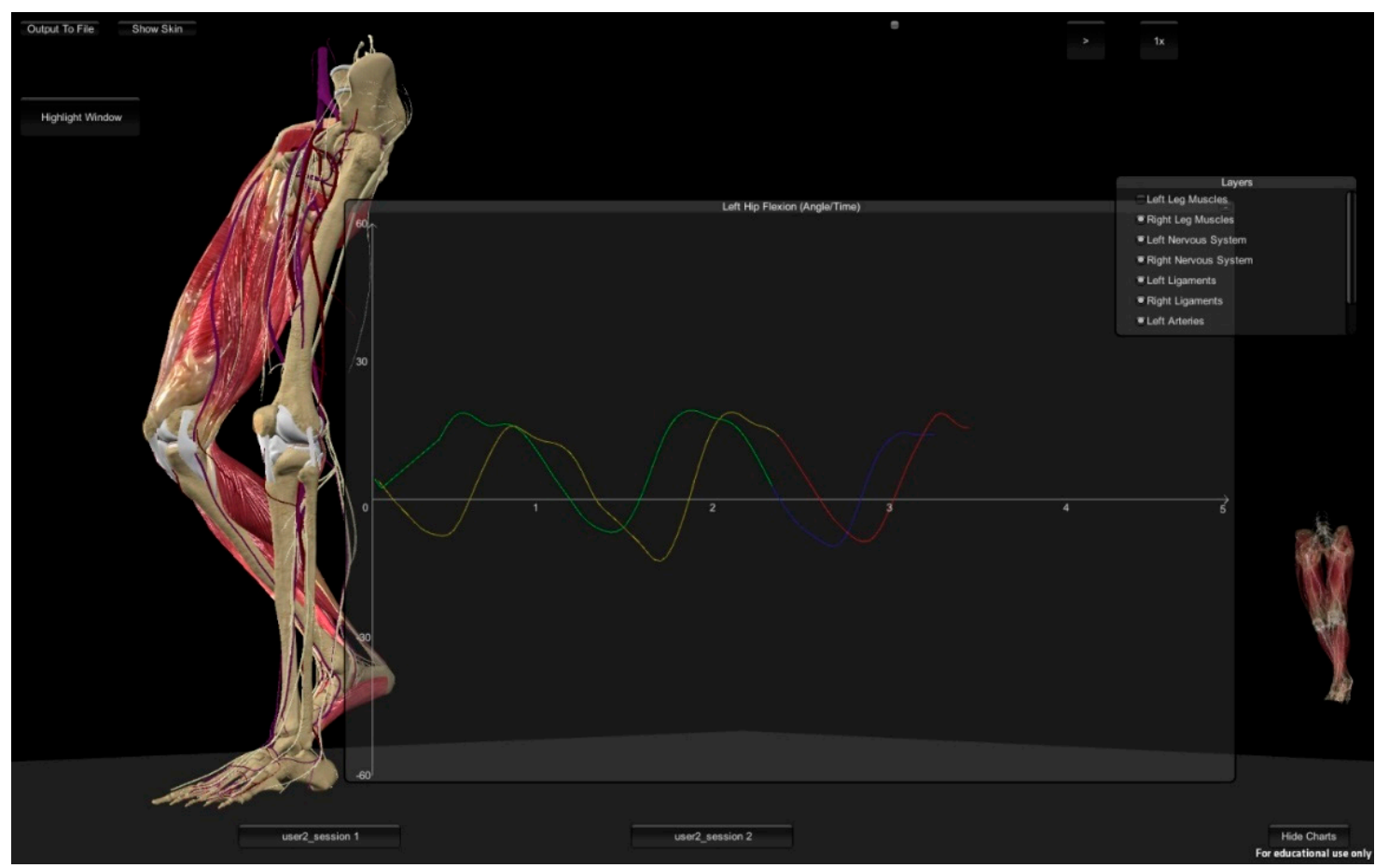

Figure 4. Comparison graphs hip flexion/extension (running live in the asynchronous virtual reality gait analysis clinic application).

\section{System Requirements}

\subsection{Hardware}

The demand for motion capturing systems is greater than ever, with a range of industries understanding the potential of such systems and its capabilities. Motion capture systems have surfaced into many cross-platform disciplines.

As previously mentioned, motion capture setups have been expensive, required specialised costly equipment, required professional setup, trained professionals, and a dedicated large space.

Rapid technological evolvement, specifically in the last decade, has rendered current large-scale production motion capture systems inexpensive (approx. £800), and it has enabled the use of marker-less motion capture, which negates the need for reflective markers on the subject and is easily accessible (utilising off-the-shelf sensors).

Whether an off-the-shelf motion-sensor device (i.e., Kinect (Microsoft ()), PS eye (Sony (C), Xtion (Asus (C) could be utilised to obtain data that can be of diagnostic value, to the clinician or the accuracy of their results, in comparison to the traditional marker-based Mo-cap system for gait analysis had not been tested prior to our work.

\subsection{Software}

Game engines are of particular interest, in contrast, to fully custom developed engines. During the exploration of existing systems and game engines regarding cost efficiency, community support, existing libraries, and ease of development and use, this work deemed that the Unity 3D engine complied with most the aforementioned characteristics. As such, the particular game-engine was employed to build upon the prototype software application. Furthermore, the particular engine allowed the development of customizable tools, and it was compatible with various types of motion capture data whilst being able to visualise high-quality 3D data in real-time. Additionally, this is a cross-platform engine, which enables the proposed software application to be easily modified to 
operate on a host of different platforms (Windows, Apple, and Android) and devices (computers, phones, and tablets).

\subsection{D/VR High Definition, Accurate Anatomical Models}

Another major consideration for the development of a VR gait analysis application is not only the acquisition of data but also their presentation to both patients and medical practitioners. The photorealistic and accurate presentation of human anatomy forms a common ground for communication for both groups, as this was revealed by previous studies [42-45]. As such, the accuracy of the provided data both in motion and visual representation could enhance further the patient/user experience and convey in a more understandable manner the complex medical issues that might hinder the patient's mobility and/or progress of rehabilitation.

For this study, we opted for an anatomical data set provided by Core Lab + Ltd. [46]. The highly detailed and accurate models offer real-time photorealistic medical 3D visualisation. Core Lab + 3D models are constructed and verified by specialised medical practitioners from relevant fields such as orthopaedic surgeons, radiologists, and general practitioners. The models' 3D meshes were further optimized for smooth real-time interaction and for use in different operating systems for VR applications, as presented in Figure 5.

The particular models consisted of more than 300 different anatomical elements including muscles, ligaments, fasciae, blood vessels, nerves, lymphatic drainage, and bones, which were developed to move and follow the leg and body motion. As such, these models enabled a photorealistic representation of a patient's gait analysis movements, which could highlight clearly the section and part of the leg that operated sub-optimally and affected overall motion.

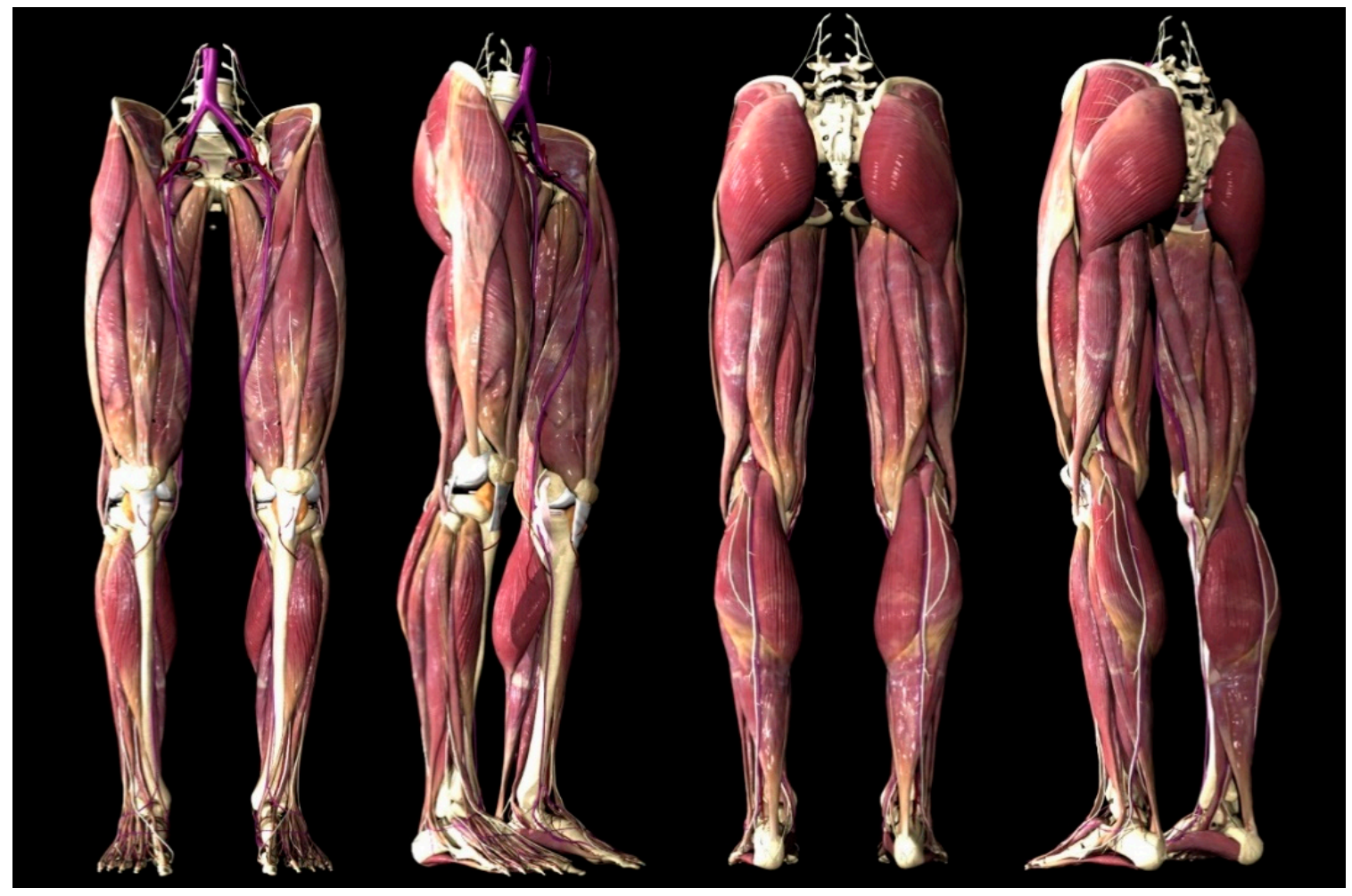

Figure 5. Optimized 3D anatomical dataset with 3D mapping applied.

The different layers of the human anatomy models could be removed or applied in real-time so as to inform the patient further regarding the different anatomical issues that he/she was encountering. This process was particularly useful as the patient was educated to understand their personal medical condition and their own circumstances. By understanding the rehabilitation rationale, the patient 
could further improve the exercise regime. At the same time, the motion data and 3D visualization offered the health professionals an instant overview of the patient's progress.

The aforementioned three elements were utilised in the development of the proposed VR gait analysis system, as presented in the following section.

\section{System Evaluation and User Feedback}

The proposed system was designed and implemented following the strict requirements of the contemporary, high-fidelity, rehabilitation systems currently used in the clinical environment. In order to ensure the proposed VR systems' compatibility with the National Health Service (NHS) requirements, the system was also tested against the high-end motion capture system available at the School of Health and Life Sciences (SHLS) at Glasgow Caledonian University (GCU). The data were retrieved simultaneously, with both systems capturing the same locomotion data, and were almost identical and within the required limits.

The study acquired the relevant ethical approval from the University's Ethics Committee. In order to achieve this, a number of experiment areas were prepared meticulously, and they followed all the current NHS guidelines for musculoskeletal rehabilitation. The process was also explained step by step to the participants, and their written and signed consent was acquired prior to the experiment.

This paper presents results of a pilot study for the user evaluation trials, designed to identify the users' technology acceptance levels. Notably, the main points of interest were user views regarding the utilization of VR for rehabilitation and the perceived ability to deploy such system in small spaces such as typical rooms in a home, hospital, or general practitioners' surgery. In addition, this work was interested in revealing any additional benefits or drawbacks that could potentially surface through the evaluation and the users' subjective feedback. The user experience and the level of technology acceptance received from this experiment would further inform this project and other future endeavours towards developing more user-friendly and cost-efficient rehabilitation systems.

For this evaluation, ten participants took part in this pilot study, $80 \%$ male, $20 \%$ female. A range of age groups was included in an attempt to minimise age bias. Fifty per cent of the participants were 25 to 29 years old. Thirty per cent of the participants were 45 years of age and over. Weight and height were also measured for each participant. The heaviest participant was $133 \mathrm{~kg}$ and the lightest was $57 \mathrm{~kg}$. The tallest person was $1.95 \mathrm{~m}$ and the shortest participant in the study was $1.64 \mathrm{~m}$.

Regardless of the height, weight, and different body types, the Mo-cap data of all the participants were collected and successfully processed into the prototype system.

It has to be noted that this paper presents a pilot study designed to identify the users' intentions towards this type of technology and rehabilitation method. Consequently, the ten participants' evaluations do not yield any statistical information, however, it provides an informative appraisal of the users' expectations of the proposed system and the system's performance.

The subjective feedback provided by the patients was recorded in two phases, namely, in the pre-test and post-test questionnaires. The following sections present the results indicative of proposed and future system requirements.

\subsection{Pre-Test Results of Users' Subjective Feedback}

This questionnaire was carried out prior to the patients being exposed to the proposed telemedicine system, before the data acquisition process (Mo-cap), presentation, and 3D demo. The objective was to try and get information from the patients without influencing their answers with the proposed research.

The first set of questions targeted the concept of telemedicine. The objective was to determine how familiar the participants were to the practice and if they had utilised it in the past. Findings indicated that even though $50 \%$ of the participants were not familiar with the term telemedicine, approximately $90 \%$ had used some form of telemedicine service previously. This shows a lack of familiarity with the term "telemedicine". Data shows that the most common form of telemedicine service used previously 
by the participants was NHS 24/Direct, which is an out-of-hours service; ninety per cent of the participants had used it previously. The NHS's symptom checker website was reported to have been used by $70 \%$ of the participants; again, this service is also considered to be an out-of-hours service for primary care. Only $20 \%$ of the participants had used the telecare monitoring services, which were related to other long-term pathologies. None of the participants had used any telemedicine service related to their gait pathology or injury. Although the patients had utilised telemedicine services, they did not know they were utilising a telemedicine system. The objective of this question was to help the patients make a connection to what they had used in the past and what the undertaken research was proposing. All the patients had used some sort of telemedicine-based service in the past; none of the services was used for their pathology related to gait-related injury or locomotion. By asking the participants about their previous experience of using telemedicine, the concept of practical telemedicine was explained, making it simpler for the participants to understand what this research was proposing. The next set of questions was designed to acquire information about the patient's history related to their pathology, appointment waiting time, and how frequently the patient visited health professionals regarding their pathology.

The most common waiting time for an initial consultation amongst the participants was 12 weeks; this was mostly to see a specialist health professional regarding their pathology. After their initial appointment, $70 \%$ of the patients were visiting the health professionals regularly every 1 to 4 weeks for the course of their treatment (Figure 6 (left)).

The next set of questions examined the length of time patients had experienced gait symptoms and explored the level of patients' familiarity with their pathology and pathology-related terminology.

According to the results, $70 \%$ of the participants experienced gait-related problems for over six years, whereas for the remainder 30\% the participant's gait abnormalities had persisted for two to five years (Figure 6 (right)).

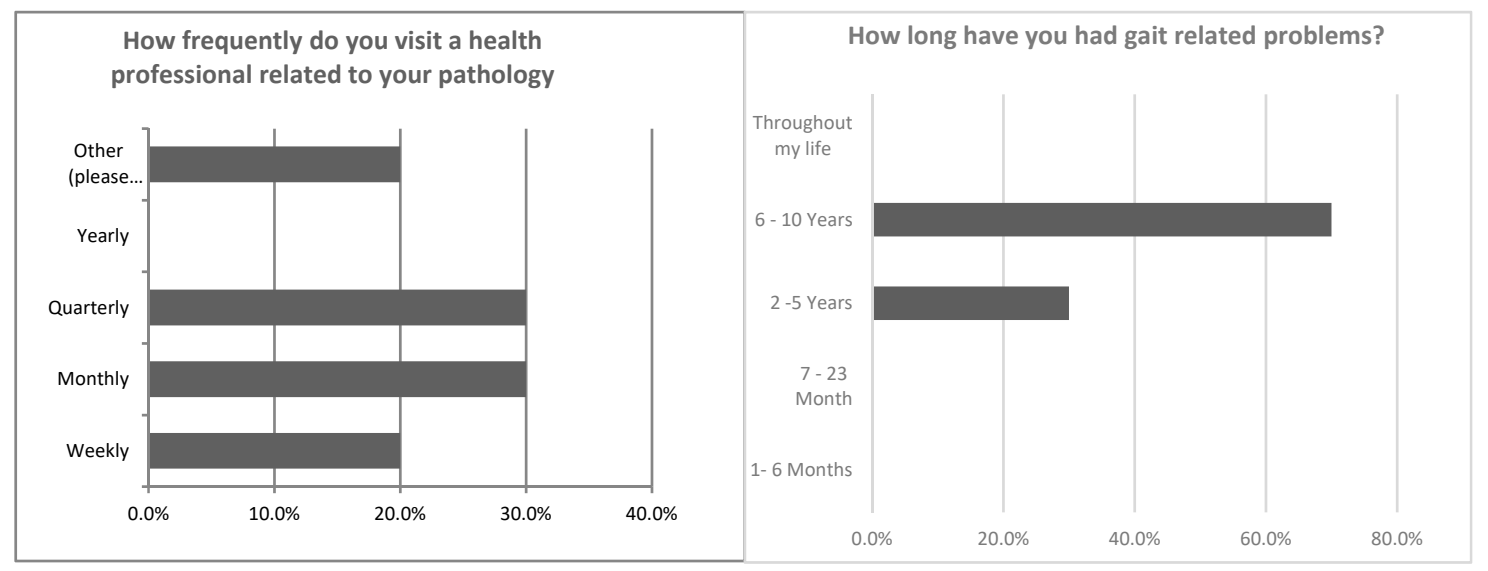

Figure 6. How frequently the patients visited health professionals (left). How long the patients had their pathologies for (right).

On questioning patients on their understanding of their pathology, $40 \%$ graded their understanding as moderate, $30 \%$ as light, and only $20 \%$ of the patients felt they understood their pathology extremely well (Figure 7 (left). Participants were further asked on their understanding of the associated pathology-related terminology. Seventy per cent of the participants felt they were not at all, slightly, or only moderately familiar (Figure 7 (right)) with such terminology. On further analysis, it became apparent that the only patients that had subjectively graded themselves as extremely familiar with their pathology or terminology were from medical and health-related educational backgrounds. Overall results highlighted that although the majority of the participants had gait-related problems for a long period of time, their understanding of the underlying pathological process or relevant 
terminology was limited. It was also evident that current interactions with the health professionals were limited in their educational value regarding the conditions under treatment
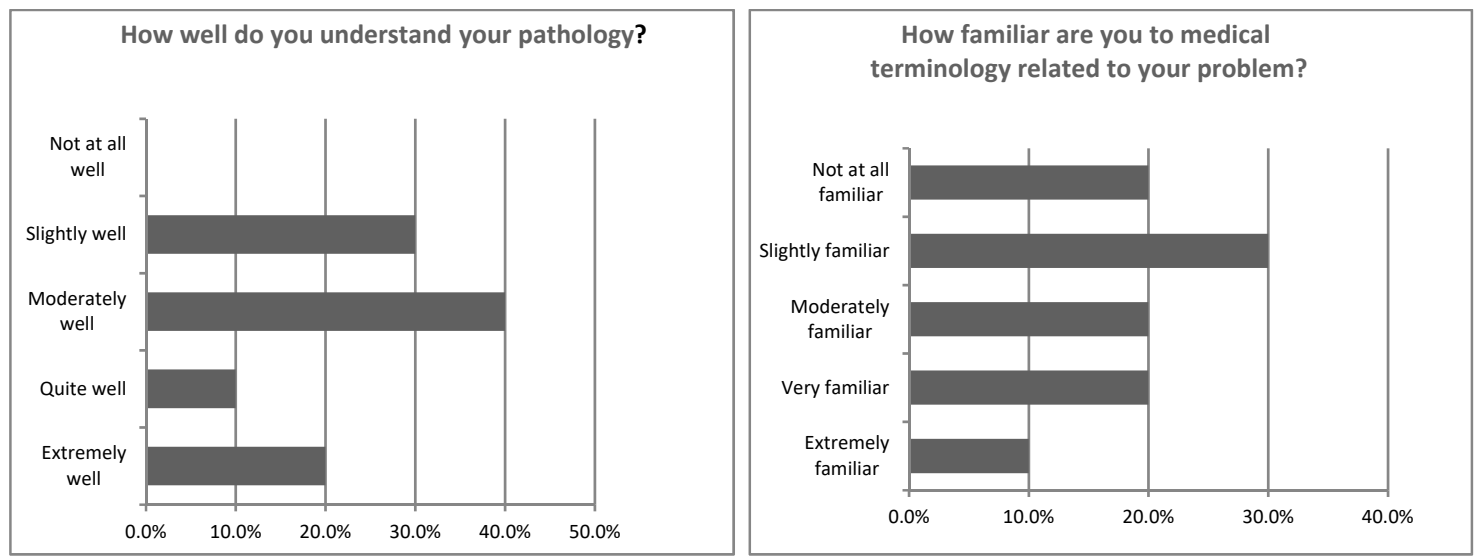

Figure 7. How well the patients understand their pathology (left). Familiarity with terminology related to pathology (right).

To this end, the next set of questions aimed to establish which techniques had been previously utilised to aid the patient's understanding of their pathology or injury, and which aspects of the information provided by the health professionals did the patients find difficult to comprehend.

Health professionals utilised verbal consultations as the main means of information sharing, and it was the main means experienced by $80 \%$ of the participants. The second most common means of conveying information was "images", and $40 \%$ of patients had some experience of this in clinical practice. Few of the participants had also experienced the use of "props" such as a skeleton model in order to visualise and understand their pathology.

3D animation or 3D visualisation techniques had not been experienced by any of the study participants in the past as aids in their understanding the pathological changes of their condition or injury (Figure 8 (left)).

Participants were further questioned whether they had difficulties during their consultations with understanding medical information. Findings revealed that $80 \%$ of the participants found understanding complicated terminology problematic, with difficulties encountered in visualising their pathology and/or injury, and a further $50 \%$ were challenged with the visualization of the underlying anatomy (Figure 8 (right)).

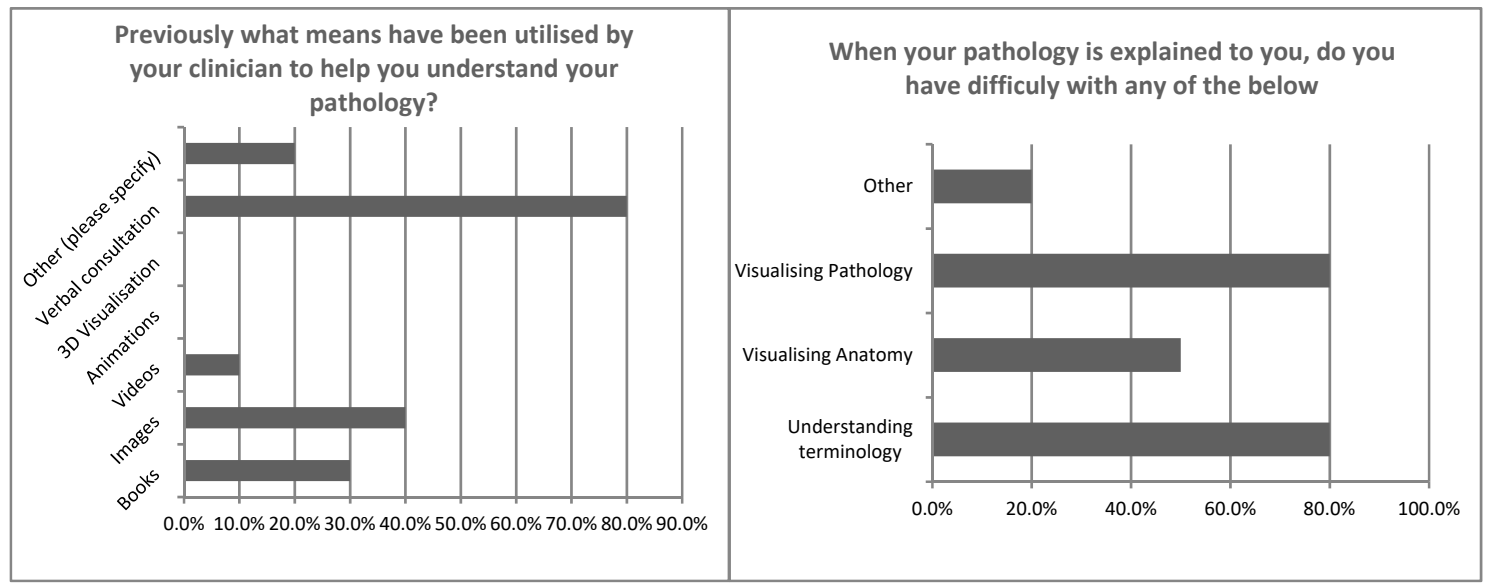

Figure 8. Previous means utilised to help understand pathology (left). Difficult aspects related to patient's pathology (right). 
It is of interest that despite $40 \%$ of the participants subjectively claiming that they understood their pathology moderately well, $80 \%$ admitted to having difficulties visualising their pathology.

The next section presents post-test user feedback, which further examines the users' intentions towards the use of emerging technologies.

\subsection{Post-Test Results of Users' Subjective Feedback}

After the data acquisition process (Mo-cap), presentation, and 3D demonstration, the users completed a post-test questionnaire. The objective was to obtain their views in regards to a telemedicine-based musculoskeletal diagnosis service, the prototype system, the data acquisition process (Mo-cap), and the method of 3D visualisation as a means of communicating patient information regarding underlying pathology.

The first set of questions explored patient views regarding the telemedicine-based musculoskeletal diagnosis service, including the prototype system that was demonstrated and presented to the participants. Participants were questioned on their views regarding the proposed system and whether they would prefer to use it (Figure 9 (left)).

Regardless of factors such as gender, age, familiarity with telemedicine, and duration of gait-related problems, findings revealed that $100 \%$ of the participants either "agreed" or "strongly agreed" that they would utilise the 3D virtual reality telemedicine service if it was implemented at their local general practitioner clinic or health center, whilst $80 \%$ either "disagreed" or "strongly disagreed" that they would prefer to utilise their current method of receiving health care rather than using this telemedicine service. These results indicated that participants had a very positive attitude towards the proposed telemedicine service. Again, $80 \%$ of the participants indicated a preference for utilizing the system independently rather than attending at a facility (Figure 9 (left)).

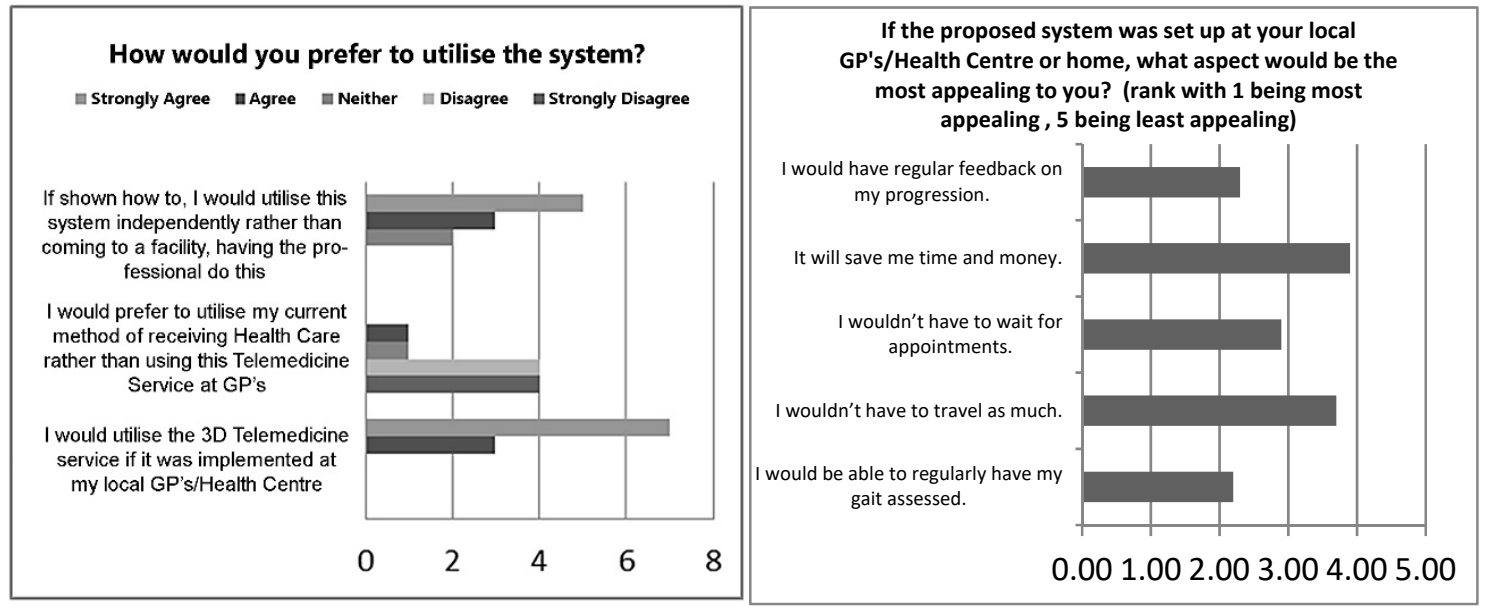

Figure 9. How and if the participant would prefer to use the system (left). What the participants found most and least appealing about the system (right).

The following questions aimed to identify which aspects of the system were considered most and least appealing. Findings revealed that with an average rating of 2.2, the most appealing factor in system implementation was the potential for regular gait assessment, followed with an average rating of 2.3 for the prospect of regular feedback regarding their progression. Results interestingly also indicated that the least appealing factor was time and money, with an average rating of 3.9 (Figure 10 (right)).

Participants were further questioned on who in their view such a system would benefit the most. Eighty per cent of the participants thought that such a system's implementation would benefit patients living in remote areas the most. Participants thought it would be least beneficial for patients who routinely did not attend appointments ((Figure 10 (left)). 
The utilisation of such a system directly from home was explored, with participants asked to indicate the most and least concerning aspect of such an endeavour (Figure 10 (right)). Participants were mostly concerned with the learning curve associated with being able to utilise the system properly $(60 \%)$, whilst the majority of participants indicated that the least concerning statement was "lack of trust in new technology and its accuracy" (80\%). Overall findings suggest that participants were willing to trust new technologies that would help them assess their gait more regularly.

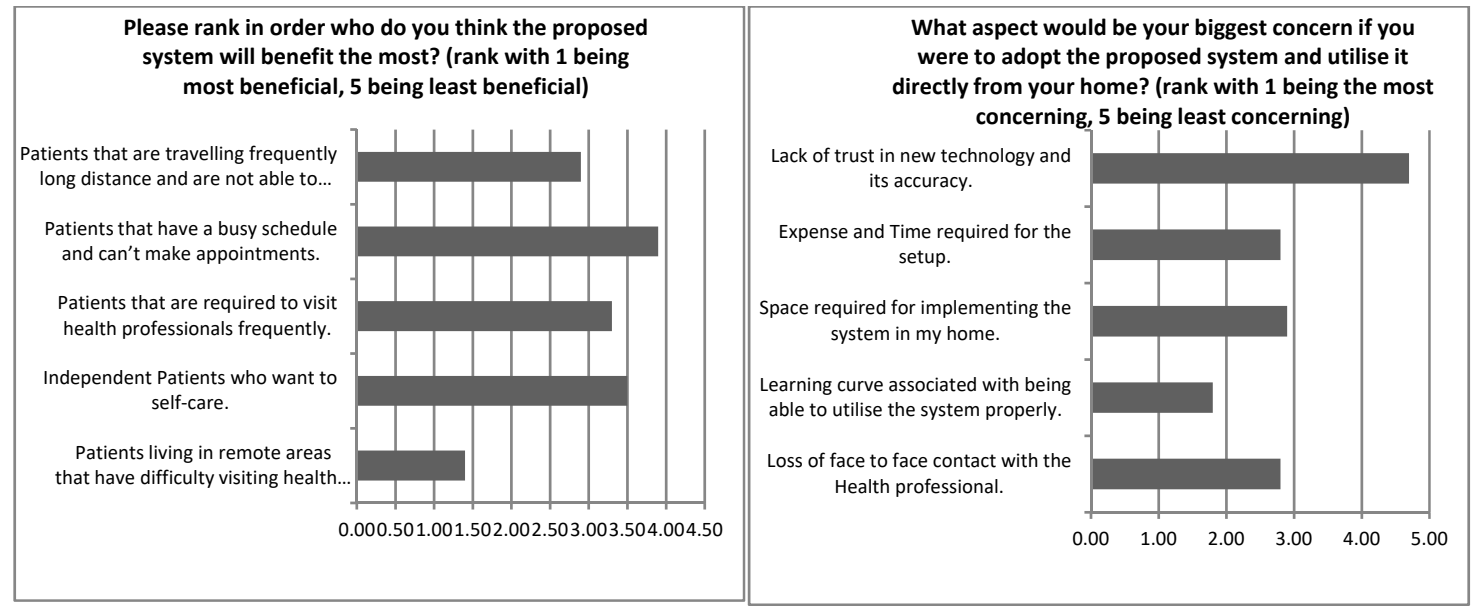

Figure 10. Perceived group of patients that would receive maximum benefit from the system (left). The most and least concerning aspects of using the system from the patient's home (right).

Participants were asked to respond to a number of statements regarding their views on their current method of receiving healthcare and their views on the new proposed telemedicine system. Findings from these results were very positive towards the proposed telemedicine system (Figure 11 (left)). This was based on average ratings, with a value of 1 being "strongly disagree" and a value of 5 being "strongly agree". With the highest average rating of $4.7,70 \%$ of the participants strongly agreed that there were more advantages than disadvantages to the new proposed system. Most of the participants "strongly disagreed" that they would carry on using their current method of receiving healthcare as they are used to the routine $(70 \%)$.

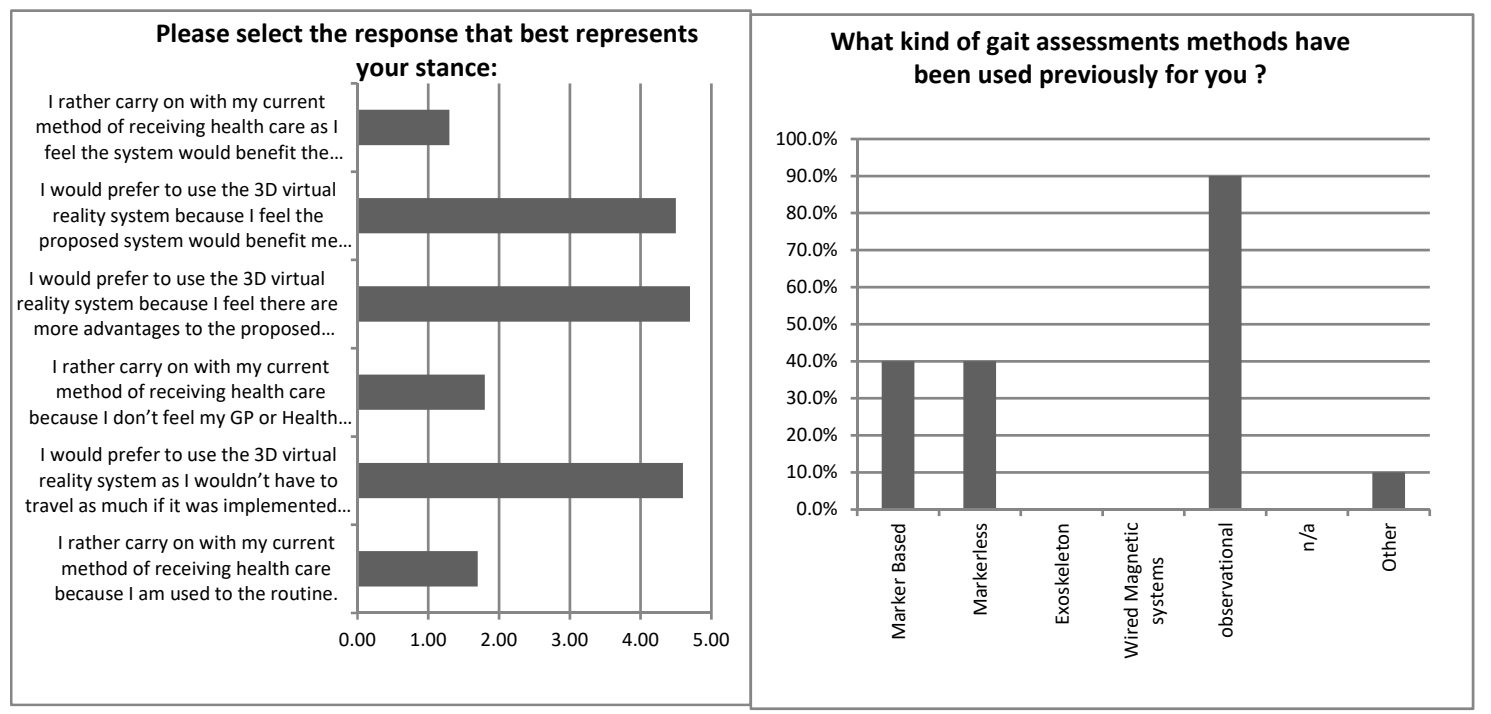

Figure 11. Willingness to use the telemedicine system (left). What kind of assessment had been used on the patient (right). 
Furthermore, the results showed that $100 \%$ of the participants either agreed or strongly agreed with the following statements:

1. "I would prefer to use the 3D virtual reality system as I wouldn't have to travel as much if it was implemented at my Local GP's or Health Centre."

2. "I would prefer to use the 3D virtual reality system because I feel there are more advantages to the proposed system than disadvantages."

3. "I would prefer to use the 3D virtual reality system because I feel the proposed system would benefit me more in comparison to the current method."

Additional interesting findings from this data included that between $80-100 \%$ of the participants either "disagreed" or "strongly disagreed" with the following statements:

4. "I would rather carry on with my current method of receiving health care because I am used to the routine."

5. "I would rather carry on with my current method of receiving health care because I don't feel my GP or Health Centre has the specialist training required to record my gait."

6. "I would rather carry on with my current method of receiving health care as I feel the system would benefit the health professionals more than me."

These findings illustrated that all participants indicated a preference for the use of the proposed system in comparison to their current method. The next set of questions focused more on the aspects of gait assessment and the methodology (Mo-cap) employed by the proposed system.

The participants were asked about their previous experience regarding gait analysis methods. Some $(60 \%)$ of the participants previously had their gait recorded. Furthermore, participants were asked about the gait assessment methods previously used to assess their gait. By far the most common (90\%) was a visual observational assessment method by the health professionals. Just under $40 \%$ used markerless and marker-based methods in the past (Figure 11 (right)). Thus, it was of interest to note that, although most of the participants had gait-related problems for over six years, nearly half of them had never had their gait recorded. Additionally, for the vast majority of participants, only observational assessments were utilised for gait analysis. As per our results, $90 \%$ of the participants considered our proposed system more useful in their gait analysis than any other system they had utilised (Figure 12 (left)). Participants were also presented with statements regarding the motion capturing process. The result showed that $100 \%$ of the participants either "agreed" or "strongly agreed" with the following statements:

1. "I found the motion capturing process straight forward and that it was not time-consuming."

2. "I liked the fact that there no wires or markers attached to me."

None of the participants found it difficult taking part in the motion capturing process. The majority of the participants liked the fact that they did not have to walk any significant distance while having their gait assessed (see Figure 12 (right)). After the data acquisition process (Mo-cap), presentation, and 3D demonstration, the users completed a post-test questionnaire. The objective was to obtain their views in regards to a telemedicine-based musculoskeletal diagnosis service, the prototype system, the data acquisition process (Mo-cap), and the method of 3D visualisation as a means of communicating patient information regarding underlying pathology.

The following sets of questions regarded the 3D VR tool (proposed system and osteoarthritis tool). Participants were asked questions regarding their thoughts on how helpful they thought the 3D VR tool was to them. The findings were extremely positive towards the 3D visualisation tools (Figure 13). 


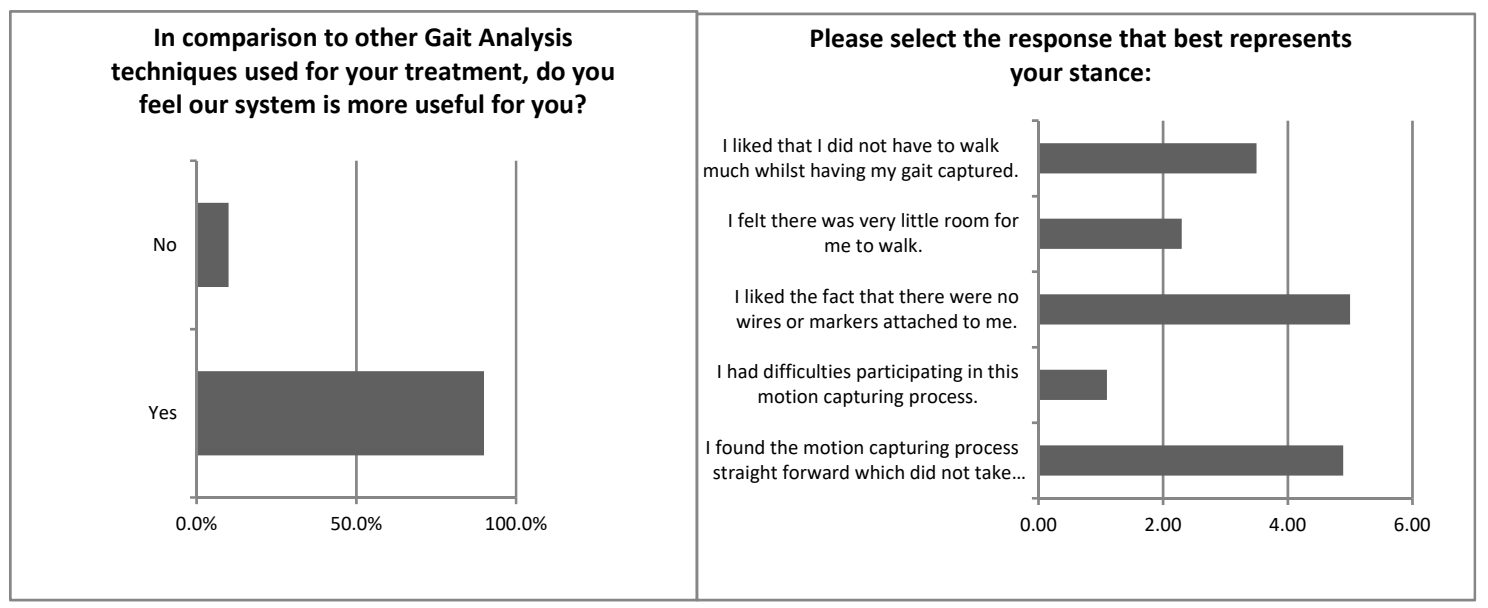

Figure 12. How useful was this system compared to other techniques (left)? Statements regarding motion capturing (Mo-cap) and patient's agreement or disagreement (right).

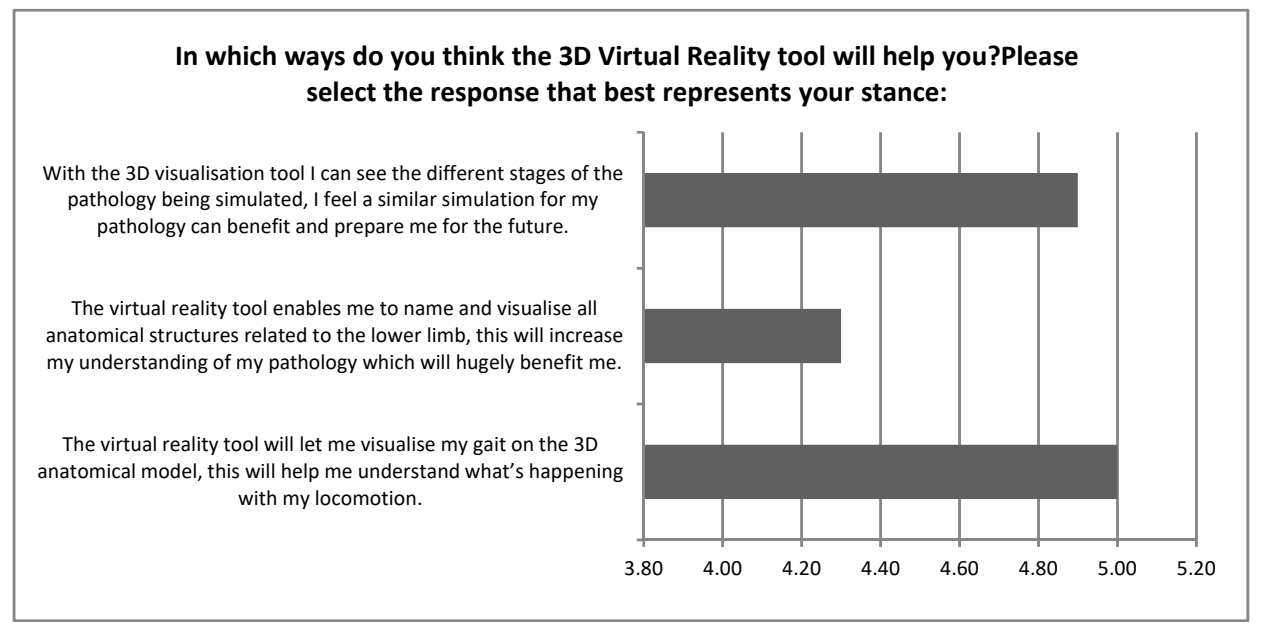

Figure 13. A graph to show in which ways the participants thought the 3D virtual reality tool will help them.

The result showed that $100 \%$ of the participants thought the VR tool (proposed system) would let them visualise their gait on a model, and help them understand what was happening with their locomotion. Additionally, $100 \%$ of the participants also thought that with VR tools (visualization regarding stages for osteoarthritis), they could visualise different stages of the pathology being simulated, and felt that a similar simulation for their own pathology could benefit them to prepare for the future. Almost 100\% of the participants either "agreed" or "strongly agreed" that the VR tool (proposed system) would help enable them to name and visualise all anatomical structures related to their lower limb, and that it would increase their understanding of their pathology, which would be hugely beneficial for them.

All the participants answered yes for the following questions:

1. "Do you think such 3D visualisation tools, would be helpful for you to understand your pathology?" (Figure 14 (left))

2. "Do you think such 3D visualisation tools could help you understand your underlying anatomy?" (Figure 14 (right))

3. "In comparison to other methods used to help you understand about your pathology, do you feel the 3D medical visualisation method shown today was better?" (Figure 15 (left)) 


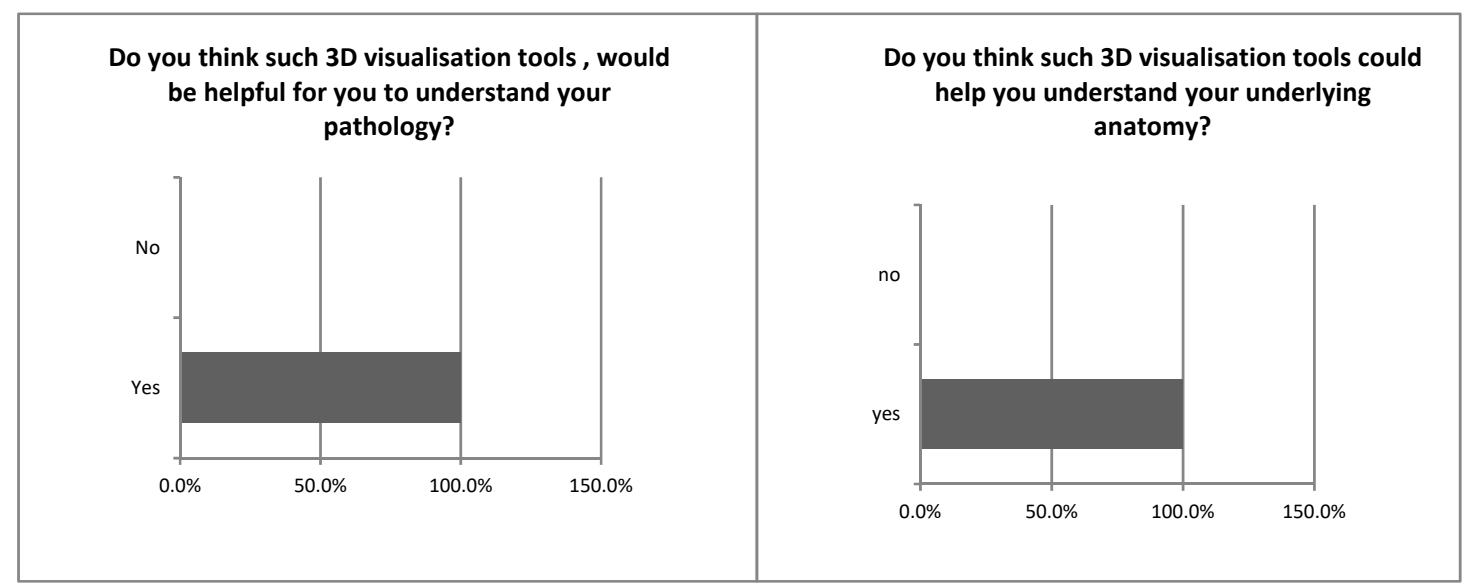

Figure 14. Do you think such 3D visualisation tools would be helpful to understand your pathology (left)? Do you think such 3D visualisation tools could help you understand your underlying anatomy (right)?

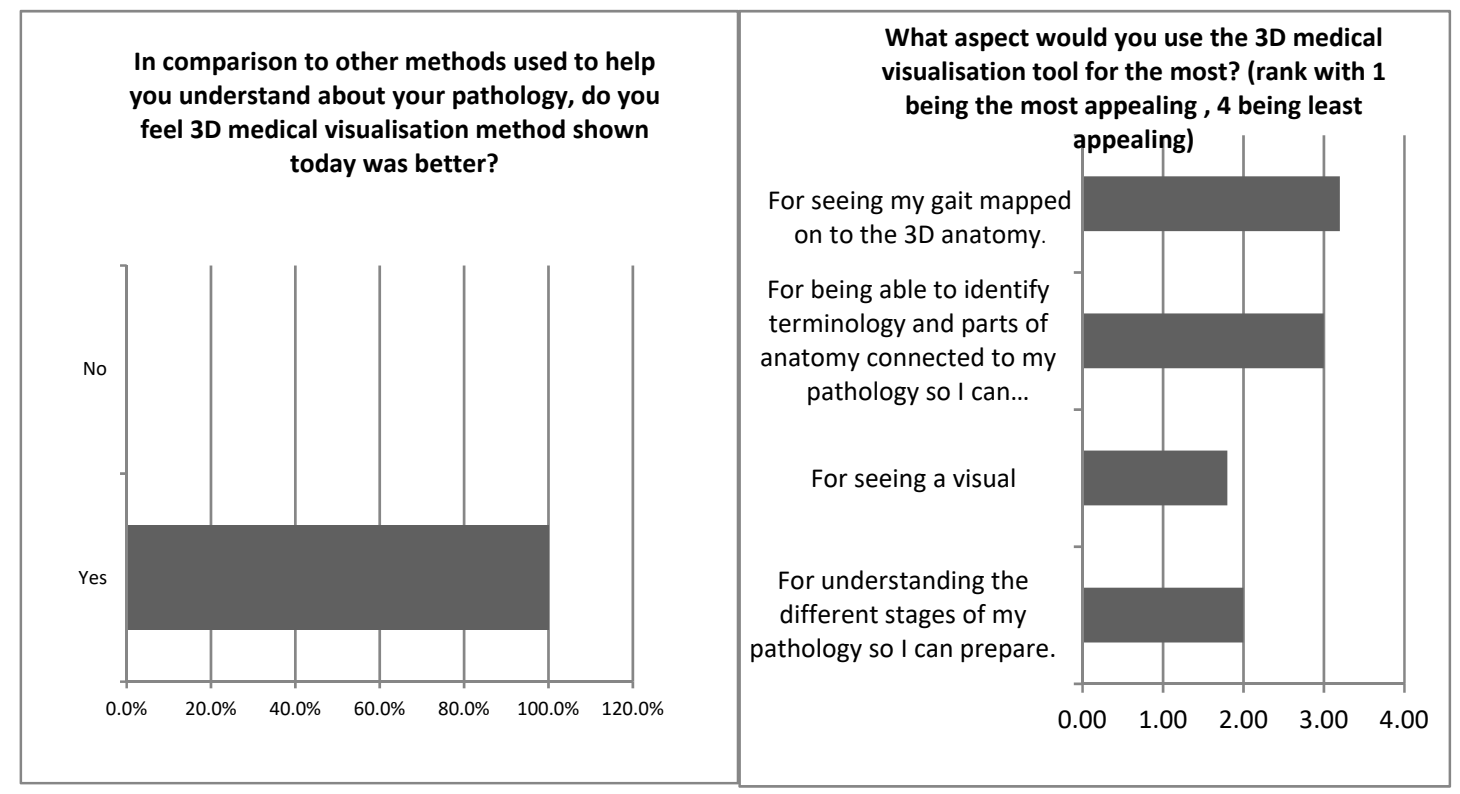

Figure 15. In comparison to other methods used to help you understand about your pathology, do you feel 3D medical visualisation method shown today was better (left)? What aspect would you use the $3 \mathrm{D}$ visualisation tool for the most (right)?

The participants were additionally asked which specific aspect of the VR tools they would utilise the most. It was evident that participants wanted to use the system mostly for mapping their own gait on the 3D anatomical dataset for visualisation and to identify terminology and parts of anatomy connected to their pathology so they can understand health professionals and increase their overall disease understanding. (Figure 14 (right)).

Through the evaluation process, it was highlighted by the participants that the technological advances used by the proposed system provided an efficient, informative, and enjoyable process. One of the major aspects was the ability to scan their gait motion in close proximity with discreet equipment. This was in direct contrast to the intimidating plethora of equipment typically hanging from the ceiling gantry of large-scale facilities and the multiple cameras/sensors staged across a long-distance corridor.

Another major aspect that was tackled with the use of the proposed system and the technologies employed was the markerless MO-CAP capability of the sensors. This was again in divergence to 
the white/reflective small spheres attached to patient joints before gait analysis and rehabilitation sessions. These markers tend to require precise positioning and fasting of any loose clothing, whilst the human factor of positioning the spheres could result in distorted results. This was emphasized as a major annoyance issue by the patients, who in some cases were unwilling to go through the process. The markerless technology embedded in the proposed system does not require any spheres or physical object or interaction between the staff and the patient. Furthermore, because the whole body is scanned in every step by the sensors, the points of interest for each joint are calculated by human motion and not by any additional artefact attachment. As such, the results are not affected by human errors in positioning the tracking spheres.

A third technological advancement that proved equally useful and was appreciated by the users was the VR visualization and presentation of the patient's gait motion and analysis. This was deemed by the users as an essential part of any future gait analysis and rehabilitation system, as it helps in educating and informing the patients about their anatomical underlying issues and the progress of their rehabilitation.

\section{Conclusions and Future Work}

This paper presented current issues regarding gait analysis in the clinical setting and the significance of its use in further understanding and rehabilitating various pathologies. Additionally, it explored limitations of the current systems with a view to map the design requirements for a prototype telemedicine-based asynchronous virtual reality gait analysis clinic. The prototype system was tested for result accuracy and fidelity in comparison to current clinically used methods with promising outcomes. Further development concentrated on the system's evaluation with regards to user experience, as the latter can present significant challenges in the incorporation of new, emerging technologies on the existing health provisions/services. To this end, pre- and post-test questionnaires were developed in order to acquire user/patient views towards technology acceptance of the proposed system. In turn, data analysis was performed with an indicative group of 10 users who were all experiencing or had experienced typical rehabilitation methods and gait analyses offered by public hospitals and/or private clinical facilities. As this was a pilot study, the acquired data were not statistically significant, yet provided an informative appraisal of the system's performance towards user experience and technology acceptance.

Overall, the users' experience presented positive results and evident preference for the adoption of the proposed prototype system within the National Health Service provision. The technological elements and advances employed, in conjunction with the bespoke developed software, could enable a cost-efficient and patient-accessible method of gait analysis and rehabilitation. The participants' feedback highlights the simplicity and efficiency of the system in contrast to the users' experience of the current state of the art facilities that are time-consuming and difficult to use. These positive user responses further informed future research plans for the development of a bespoke hardware and software VR system that could be deployed and operated directly from the patient's home whilst monitored by the healthcare professionals.

Concluding, the overall experiment offered an intriguing insight into the requirements of future rehabilitation applications. To this end, it was evident that patients were welcoming the acquisition of medical information regarding their pathology. Yet, this should be presented in a simplified yet visually enticing manner. This type of information could further support the patients' consent process and empower the patients to understand the methods and the required outcomes of their rehabilitation.

Author Contributions: Conceptualization, M.S.K. and V.C.; methodology, M.S.K.; Software, M.S.K.; validation, S.S., M.S.K. and V.C.; formal analysis, M.S.K.; investigation, M.S.K.; resources, S.S.; data curation, V.C.; writing—original draft preparation, M.S.K.; writing—review and editing, V.C.; visualization, M.S.K.; V.C. and S.S.; supervision, V.C.; project administration, V.C.; internal funding acquisition, M.S.K. and V.C.

Funding: This research received no external funding.

Conflicts of Interest: The authors declare no conflict of interest. 


\section{References}

1. Sanders, S. National Life Tables, UK: 2014 to 2016; Office for National Statistics: Newport, UK, 2018.

2. Storey, A. Living longer - How Our Population is Changing and Why It Matters; Office for National Statistics: Newport, UK, 2018.

3. Statistical Digest of Rural England. Available online: https://www.gov.uk/government/statistics/statisticaldigest-of-rural-england (accessed on 20 January 2019).

4. Pirker, W.; Katzenschlager, R. Gait disorders in adults and the elderly: A clinical guide. Wien. Klin. Wochenschr. 2017, 129, 81-95. [CrossRef] [PubMed]

5. Van Dijsseldonk, R.B.; De Jong, L.A.F.; Groen, B.E.; Vos-Van Der Hulst, M.; Geurts, A.C.H.; Keijsers, N.L.W. Gait stability training in a virtual environment improves gait and dynamic balance capacity in incomplete spinal cord injury patients. Front. Neurol. J. 2018, 9, 963. [CrossRef] [PubMed]

6. Trew, M.; Everett, T. Human Movement: An Introductory Text, 4th ed.; Churchill Livingstone: London, UK, 2001.

7. Corazza, S.; Mündermann, L.; Chaudhari, M.; Demattio, T.; Cobelli, C.; Andriacchi, T.P. A markerless motion capture system to study musculoskeletal biomechanics: visual hull and simulated annealing approach. Ann. Biomed. Eng. 2006, 34, 1019-1029. [CrossRef] [PubMed]

8. Bálint, G.; Dezso, Z.; Hunka, A.; Lenti, J.; Loványi, I. Motion capture vs traditional medical examinations. In Proceedings of the 3rd International Conference on Sciences of Electronics, Technologies of Information and Telecommunications, Sousse, Tunisia, 27-31 March 2005.

9. Kertesz, Z.; Lovainyi, I. 3D motion capture methods for pathological and non-pathological human motion analysis. In Proceedings of the 2nd International Conference on Information \& Communication Technologies, Damascus, Syria, 24-28 April 2006; pp. 1062-1067.

10. Baker, R. Gait analysis methods in rehabilitation. J. Neuroeng. Rehabil. 2006, 3, 4. [CrossRef] [PubMed]

11. Age UK. Policy Position-Telehealth and Telecare (UK). Available online: https://www.ageuk.org.uk/ globalassets/age-uk/documents/policy-positions/care-and-support/ppp_telehealth_and_telecare_uk. pdf (accessed on 20 January 2019).

12. National Telehealth and Telecare Delivery Plan for Scotland to 2015; NHS Scotland: Edinburgh, UK, 2012.

13. Whoriskey, M. Technology Enabled Care Supporting Service Transformation Delivery Plan 2018/19. In Government Report: Digital Health and Care Scotland; The Scottish Government: Edinburgh, UK, 2018.

14. NHS Commissioning Assembly. Technology Enabled Care Services. Available online: https:/ /www.england. nhs.uk/wp-content/uploads/2014/12/TECS_FinalDraft_0901.pdf (accessed on 20 January 2019).

15. Khan, M.S.; Chan, W.; Charissis, V.; Harrison, D.K.; Sakellariou, S. Development and evaluation of prototype virtual reality telemedicine system for asynchronous gait analysis. In Proceedings of the 8th International Conference on Appropriate Healthcare Technologies for Low Resource Settings, London, UK, 17-18 September 2014; p. 18.

16. Khan, S.; Charissis, V.; Harrison, D.; Sakellariou, S.; Chan, W. Asynchronous telemedicine diagnosis of musculoskeletal injuries through a prototype interface in virtual reality environment. In Virtual, Augmented and Mixed Reality. Systems and Applications; Shumaker, R., Ed.; Springer: Berlin/Heidelberg, Germany, 2013.

17. Whittle, M.W. An Introduction to Gait Analysis, 4th ed.; Butterworth-Heinemann: Oxford, UK, 2007.

18. Kale, A.; Cuntoor, N.; Yegnanarayana, B.; Rajagopalan, A.N.; Chellappa, R. Gait analysis for human identification. In Audio- and Video-Based Biometric Person Authentication; Kittler, J., Nixon, M.S., Eds.; Springer: Berlin/Heidelberg, Germay, 2003; Volume 268, pp. 706-714.

19. Luo, $\mathrm{H} . ; \mathrm{Wu}, \mathrm{D}$. A remote markerless human gait tracking for e-healthcare based on content-aware wireless multimedia communications. IEEE Wirel. Commun. 2010, 17, 44-50. [CrossRef]

20. Furniss, M. Motion capture. In Proceedings of the Media in Transition Conference at MIT, Cambridge, MA, USA, 8 October 1999.

21. Moeslund, T.B.; Hilton, A.; Krüger, V. A survey of advances in vision-based human motion capture and analysis. Comput. Vis. Image Underst. 2006, 104, 90-126. [CrossRef]

22. Gleicher, M.; Ferrier, N. Evaluating video-based motion capture. In Proceedings of the Computer Animation 2002, Washington, DC, USA, 19-21 June 2002; pp. 75-80.

23. Perry, J. Gait Analysis: Normal and Pathological Function, 2nd ed.; Slack Firm: Vancouver, BC, Canada, 2010.

24. Walking Abnormalities. Available online: https://www.ncbi.nlm.nih.gov/gtr/conditions/C0427149/ (accessed on 22 January 2019). 
25. Stanford Medicine. Gait Abnormalities. Available online: https://stanfordmedicine25.stanford.edu/the25/ gait.html/ (accessed on 22 January 2019).

26. Salzman, B. Gait and balance disorders in older adults. Am. Fam. Physician 2010, 82, 61-68. [PubMed]

27. Public and Patient Guide: Hip Replacement Edition. 2017. Available online: http:/ / www.njrreports.org.uk/ Portals/0/PDFdownloads/NJR\%2014th\%20PPG\%20Hips.pdf (accessed on 22 January 2019).

28. Moore, K.L.; Dalley, A.F.; Agur, A.M.R. Clinically Oriented Anatomy, 6th ed.; Lippincott Williams \& Wilkins: Philadelphia, PA, USA, 2010.

29. Marieb, E.N.; Hoehn, K. Human Anatomy \& Physiology, 7th ed.; Pearson Benjamin Cummings: San Francisco, CA, USA, 2007.

30. Barrett, E.M. The impact of rheumatoid arthritis on employment status in the early years of disease: A UK community-based study. Rheumatology 2000, 39, 1403-1409. [CrossRef] [PubMed]

31. Tins, B.J.; Garton, M.; Cassar-Pullicino, V.N.; Tyrrell, P.N.M.; Lalam, R.; Singh, J. Stress fracture of the pelvis and lower limbs including atypical femoral fractures-A review. Insights Imaging 2015, 6, 97-110. [CrossRef]

32. Muro-de-la-Herran, A.; Garcia-Zapirain, B.; Mendez-Zorrilla, A. Gait analysis methods: An overview of wearable and non-wearable systems, highlighting clinical applications. Sensors 2014, 14, 3362-3394. [CrossRef]

33. Sturman, D.J. A brief history of motion capture for computer character animation. In Proceedings of the Annual Conference of Special Interest Group on Computer GRAPHics and Interactive Techniques, Orlando, FL, USA, 24-29 July 1994.

34. Desloovere, K.; Wong, P.; Wings, L.; Callewaert, S.B.; Vandenneucker, H.; Leardini, A. Range of motion and repeatability of knee kinematics for 11 clinically relevant motor tasks. Gait Posture 2010, 32, 597-602. [CrossRef] [PubMed]

35. Mitchell, G.; Clarke, A. Capturing and visualising playground games and performance: A wii and kinect based motion capture system. In Proceedings of the 2011 international conference on Electronic Visualisation and the Arts, London, UK, 6-8 July 2011.

36. Zhou, H.; Hu, H. Human motion tracking for rehabilitation-A survey. Biomed. Signal Process. Control 2008, 3, 1-18. [CrossRef]

37. Foxlin, E.; Harrington, M.; Pfeifer, G. Constellation: A wide-range wireless motion- tracking system for augmented reality and virtual set applications. In Proceedings of the Annual Conference of Special Interest Group on Computer GRAPHics and Interactive Techniques, Orlando, FL, USA, 19-24 July 1998.

38. Noonan, D.P.; Mountney, P.; Elson, D.S.; Darzi, A. A stereoscopic fibroscope for camera motion and 3D depth recovery during Minimally Invasive Surgery. In Proceedings of the 2009 IEEE International Conference on Robotics and Automation, Kobe, Japan, 12-17 May 2009; pp. 4463-4468.

39. Stone, E.E.; Skubic, M. Passive in-home measurement of stride-to-stride gait variability comparing vision and Kinect sensing. In Proceedings of the 23rd Annual International Conference of the IEEE Engineering in Medicine and Biology Society, Istanbul, Turkey, 25-28 October 2001; pp. 6491-6494.

40. Attygalle, S.; Duff, M.; Rikakis, T.; He, J.H.J. Low-cost, at-home assessment system with Wii Remote based motion capture. In Proceedings of the 2008 Virtual Rehabilitation, Vancouver, BC, Canada, 25 August 2008.

41. Sundaresan, A.; Chellappa, R. Markerless motion capture using multiple cameras 1: Introduction. In Proceedings of the Computer Vision for Interactive and Intelligent Environment 2005, Lexington, KY, USA, 17-18 November 2005.

42. Lin, Q.; Xu, Z.; Li, B.; Baucom, R.; Poulose, B.; Landman, B.A.; Bodenheimer, R.E. Immersive virtual reality for visualization of abdominal CT. In Proceedings of the SPIE Medical Imaging 2013, Lake Buena Vista, FL, USA, 10-12 February 2013.

43. Ward, B.M.; Charissis, V.; Rowley, D.; Anderson, P.; Brady, L. An evaluation of prototype VR medical training environment: applied surgical anatomy training for malignant breast disease. J. Stud. Health Technol. Informa 2008, 132, 500-505.

44. Sakellariou, S.; Charissis, V.; Grant, S.; Turner, J.; Kelly, D.; Christomanos, C. Virtual reality environment as knowledge enhancement tool for musculoskeletal pathology. In Virtual and Mixed Reality-Systems and Applications; Lecture Notes in Computer Science; Schumaker, J.R., Ed.; Springer: Berlin/Heidelberg, Germany, 2011; Volume 6774, pp. 54-63. 
45. Collins, M.K.; Ding, V.Y.; Ball, R.L.; Dolce, D.L.; Henderson, J.M.; Halpern, C.H. Novel application of virtual reality in patient engagement for deep brain stimulation: A pilot study. Brain Stimul. J. 2018, 11, 935-937. [CrossRef] [PubMed]

46. Core Lab +, Core Lab +: 3D Medical. Available online: https://www.corelabplus.co.uk/cl-medical (accessed on 20 January 2019). 